\title{
An optical method for rapid examination of check development in decorative plywood panels
}

\author{
Michael David Burnard ${ }^{1,2,3} \cdot$ Lech Muszyński $^{4} \cdot$ Scott Leavengood $^{4} \cdot$ Lisa Ganio $^{5}$
}

Received: 2 March 2017 / Published online: 15 June 2018

(c) The Author(s) 2018

\begin{abstract}
Common methods for assessment of surface checking in decorative plywood panels rely on manual handling and visual inspection of specimens, a laborious procedure practically limiting the number of materials and variables that may be considered within one project. In this study, a new automated optical method for detection and measurement of checks has been developed. This method was based on the digital image correlation principle, which allowed identification of checks as small as $0.2 \mathrm{~mm}$ wide and $1 \mathrm{~mm}$ long. Continuous measurement allowed reliable check counts, and measurement of check dimensions as they develop during exposure to drying conditions. A check severity index has been proposed. The method has been validated in exposure tests conducted in harsh but realistic conditions, to increase the likelihood of checking and reduce the test duration to $4 \mathrm{~h}$. In addition, an innovative test setup allowed near simultaneous monitoring of check development in up to 48 panel specimens sized $30 \times 30 \mathrm{~cm}$. The efficiency of the method allows studies to examine an unprecedented number of treatments and replicates.
\end{abstract}

\section{Introduction}

Decorative hardwood plywood panels are laminated woodbased composite products commonly used in applications where quality appearance is critical (e.g., in cabinetry, furniture, fixtures, and wall and ceiling panels). The panels typically consist of a core material overlaid with decorative face and back materials, which are often thin hardwood veneers. Common core materials are medium density fibreboard (MDF), particleboard (PB), plywood (PW, also referred to as veneer core), and combination cores (typically softwood veneer with a thin layer of MDF on the front and back). Common adhesives used to bond the decorative veneers to

Michael David Burnard

michael.burnard@iam.upr.si

1 Andrej Marušič Institute, University of Primorska, Muzejski $\operatorname{trg}$ 2, 6000 Koper, Slovenia

2 Abelium, d.o.o., Kajuhovu 90, 1000 Ljubljana, Slovenia

3 InnoRenew CoE, Livade 6, 6310 Izola, Slovenia

4 Department of Wood Science and Engineering, College of Forestry, Oregon State University, 119 Richardson Hall, Corvallis, OR 97331, USA

5 Department of Forest Ecosystems and Society, College of Forestry, Oregon State University, 321 Richardson Hall, Corvallis, OR 97331, USA the core include urea formaldehyde (UF) based adhesives, soy-based adhesives (Soy), and polyvinyl acetate (PVA) based adhesives.

Decorative veneers are produced from a variety of species either by rotary peeling, or various slicing methods (e.g., plain slicing, half-round slicing). Veneers used for faces are typically those considered to be of the best appearance, while backs may have lower grade appearance. In uses where appearance is most critical, any defects in the face veneer are undesirable and often lead to costly customer complaints. One of the most common defects are surface checks induced by rapid drying in low humidity environments. Since manufacturers cannot control environmental conditions after product installation (e.g., after cabinets have been installed in a house) their attention is focused on the manufacturing parameters that can eliminate or mitigate checking.

\subsection{Veneer checking}

ASTM D9 Standard Terminology Relating to Wood and Wood-Based Products provides a generic definition of a check in wood as "a separation of the wood along the fiber direction that usually extends across the rings of annual growth commonly resulting from stresses set up in the wood during seasoning." Seasoning, in this sense, means drying that occurs naturally or in kilns (ASTM International 
1999). The mechanism of development of drying stresses and checking has been extensively researched in the context of commercial drying of sawn lumber (Felix and Morlier 1992; Mårtensson and Svensson 1997; Ormarsson et al. 1999; Svensson and Martensson 2002). The principal cause of checking in hardwood plywood face veneers is variable shrinkage rates and moisture content differences between the drying face veneer and the moist core material of the product (Gilmore and Hanover 1990; Forbes 1997; Schramm 2003; Cassens and Leng 2003; Christiansen and Knaebe 2004; Leavengood et al. 2011). Checks occur when the tension stresses in the drying face veneer exceed the ultimate tensile strength perpendicular to the grain. In addition, wood composites, such as particleboard, plywood or medium density fiberboard used for decorative plywood cores, tend to shrink less than solid wood or veneers perpendicular to the grain (Suchsland 2004), which contributes to the buildup of drying stresses on the surface and may lead to more severe checking.

It is important to note that the moisture gradient and the related stress gradient build gradually during exposure to drying conditions, reach a peak and then gradually decrease until the panel reaches equilibrium with the environment. Checks are most likely to appear as both gradients increase in the early stages of the exposure and reach their peak visibility when the gradients are most severe before gradually closing as the moisture gradients decrease (Svensson and Martensson 2002). Once formed, the checks may remain visible if the new equilibrium conditions are drier than the initial conditions. Even closed checks may remain visible, especially if contaminated.

\subsection{Methods for check detection and measurement}

The appearance of checks and their dimensions may vary substantially from panel to panel and may range from a collection of minute checks barely visible to the naked eye to long and extended checks, which are immediately apparent to the eye (Holcombe 1952). However, neither ASTM D1038 (Standard Terminology Relating to Veneer and Plywood) nor the decorative hardwood plywood industry provide a definition specific to face veneer checking or specific size threshold at which a minute split is considered a check that may trigger a formal complaint. In the absence of a standard definition, individual researchers propose their own check qualification criteria in terms of minimum qualifying width or length of a check (Batey 1955; Cassens and Leng 2003). Batey (1955) measured the smallest checks plainly visible in clear light using a 40x tube microscope and found they were approximately $0.025 \mathrm{~mm}$ wide and considered this the lower limit of what could be counted as a check. Cassens and Leng (2003) counted separations along the grain that were plainly visible using a 16x scaled loupe as checks.
Although it is generally understood that check severity refers to the number and magnitude of detectable checks present in a reference area, no standard definition or quantitative metric has been proposed to date. The definitions and metrics used in previous studies focus on check count and reflect the fundamental difficulties of commonly used measurement methods relying on manual handling and visual inspection of panels (Batey 1955; Cassens and Leng 2003; Leavengood et al. 2011). Batey (1955) proposed a method in which panels were inspected with an unaided eye through a square grid of horizontal and vertical lines spaced $2.54 \mathrm{~cm}$ apart on the test specimen. Surface checks were counted one time for each line they crossed. For instance, a long check crossing three grid lines would be counted three times. Cassens and Leng (2003) enhanced this method by using a 16x scaled loupe to search for checks. These manual/ visual methods are laborious and time-consuming effectively limiting the number of check characteristics, such as number of checks, length, and width, that can be determined and the quantity of panels that can be examined in any one study even by a skillful and experienced investigator. They practically preclude continuous monitoring of multiple checks as they develop, and check characteristics captured at an isolated point in time provide little information about the dynamics of check development. Check dimensions may and do change substantially during inspection or while waiting to be examined. Using manual/visual methods, capturing the peak check development for every specimen in a large collection of panels is extremely challenging if at all possible. Some checks may even close prior to being counted and/or measured (Holcombe 1952; Cassens and Leng 2003; Leavengood et al. 2011). Historically, these limitations have adversely impacted the number of factors such as core type, veneer thickness, adhesive, etc., that could be considered in any individual study.

Kang et al. (2011) demonstrated that full-field optical strain measurement techniques based on the digital image correlation (DIC) principle developed by Sutton and Chao (1988) can be successfully used to detect and characterize check development in wood composites. In their proof-ofconcept study, Kang et al. (2011) analyzed full field strain maps measured on the surface of drying $100 \times 76 \mathrm{~mm}$ specimens of oak veneer bonded to a rigid background and noticed characteristic peak-and-valley patterns in strain measurements near checks (Fig. 1). Although surface checks constitute discontinuities, for which, in theory, strains cannot be properly defined, the DIC algorithm routinely interprets small checks as regions with characteristic strain spikes, which are relatively easy to detect and isolate (e.g., by a threshold procedure). Kang et al. (2011) suggested that these signature peaks and valleys patterns could be used for automatic identification and characterization of surface veneer checks. They also proposed that 

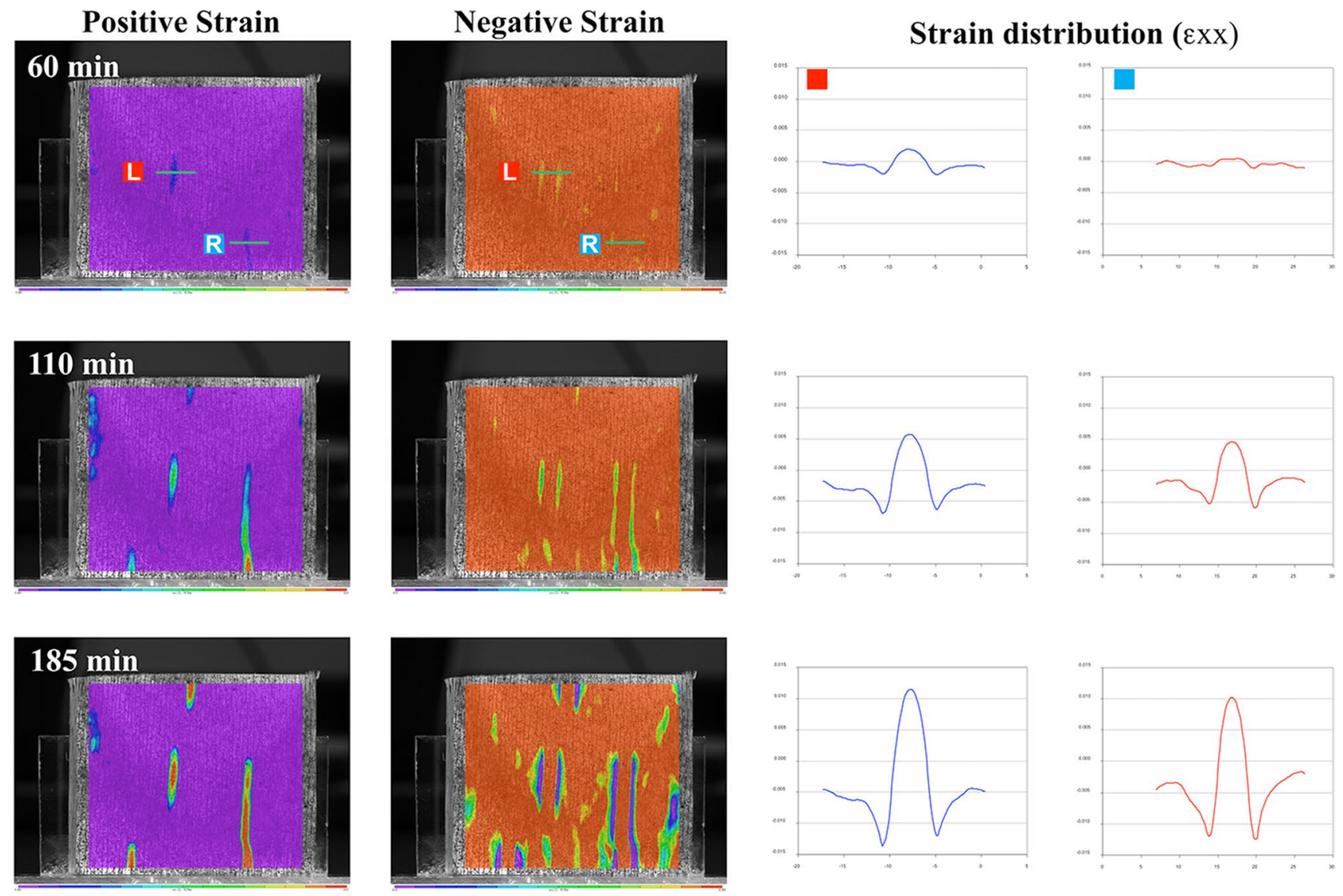

Fig. 1 Signature strain pattern observed in the proof-of-concept study by Kang et al. (2006)

the associated surface point displacement data could be used to provide size estimates of check characteristics as they develop (Kang et al. 2011).

Other DIC-based techniques have been used to detect and measure crack-tip propagation and crack characteristics in fracture testing (Rethore et al. 2008, Helm 2008; Alam et al. 2012). In these techniques, the attention is focused on an individual crack, however, they would not allow for a simultaneous measurement of multiple cracks occurring on the same surface. Non-optical methods, such as those using vibrometry, are well suited for detecting and measuring interior damage (such as cracks), but may not be suitable for measuring and characterizing surface cracks or multiple cracks (c.f. Fan and Qiao 2011).

\subsection{Objectives}

The overall objective of this research was to develop an efficient method for detection and measurement of surface checks in decorative plywood specimens based on optical measurements as proposed by Kang et al. (2011). Specific objectives were to:
1. Propose clear and objective criteria to qualify visible surface features as checks in an automated procedure.

2. Develop an algorithm for automated characterization of the detected checks in terms of check counts and dimensions (i.e., check length and width).

3. Propose a quantitative check severity index based on the measured characteristics for rapid objective comparison of damage observed in various materials.

4. Develop a specimen handling system that would allow concurrent examination of check formation dynamics in a large number of panel specimens (i.e., panel counts in the hundreds).

5. Demonstrate the efficacy of the method in a pilot study.

The method described here was developed for a research project involving decorative panels with a thin sugar maple (Acer saccharum) veneer overlay where the effects of a subset of manufacturing factors commonly believed to contribute to veneer checking (including core type, adhesive, veneer thickness, and face veneer attributes) were examined. 


\section{Materials and methods}

The approach for this study was to develop a batch exposure test method for several $30 \times 30 \mathrm{~cm}$ decorative hardwood plywood panels, with automated check detection and analysis procedures. The starting point for the development of an automated method was the optical method proposed by Kang et al. (2011). Rapid automated examination of up to 48 test specimens $(30 \times 30 \mathrm{~cm})$ was possible by mounting a digital camera on an $8 \mathrm{~m}$ horizontal linear positioning track system ( 25 images of 48 test specimens in $240 \mathrm{~min}$ ). Use of relatively harsh but realistic exposure conditions enabled a substantial reduction in testing time. Finally, automated identification of checks allowed for batch processing of the data collected during the exposure tests.

\subsection{Defining qualifying checks and check intensity index (check density)}

The absence of standardized criteria for the qualification of visible surface features as checks is a serious challenge for any attempt at an objective, and preferably automated, check detection procedure. For the purpose of this study the criteria were selected based on visual examination of four decorative panels with existing checks identified by hardwood plywood professionals and their customers. These panels were examined under a microscope (20x magnification) and with a 9x scaled loupe to determine the minimum width an automated optical method would need to detect. All checks detected on these panels were greater than or equal to $0.2 \mathrm{~mm}$ in width and more than $1.0 \mathrm{~mm}$ long. Therefore, for the purpose of this study, these parameters were accepted as the smallest dimension of surface discontinuities to be qualified as checks.

In addition, a check severity index, check density (CD) was defined. This indicator captures more information about checking than previous measures, such as total check length of visible checks, check count, or derived indicators (e.g., as in Holcombe 1952). CD is expressed as the total area of all observed checks on a specimen $\left(\mathrm{A}_{\mathrm{ck}}\right.$ in $\left.\mathrm{mm}^{2}\right)$ divided by the reference surface area under examination (the region of interest) $\left(\mathrm{A}_{\mathrm{ROI}}\right.$ in $\mathrm{m}^{2}$, Eq. 1). $\mathrm{A}_{\mathrm{ROI}}$ may vary slightly from specimen to specimen if the region of interest is manually selected by the software operator during analysis.

$$
C D=\frac{A_{c k}}{A_{R O I}}\left[\frac{m m^{2}}{m^{2}}\right]
$$

\subsection{Experimental setup}

The experimental setup consisted of a climate control system enclosing an optical measurement system with a digital camera mounted on an $8 \mathrm{~m}$ horizontal linear positioning track moving over a stage on which panel specimens were arranged (Fig. 2). The setup also included a data processing unit with custom image capture and track control software. This setup allowed examination of up to $48,30 \times 30 \mathrm{~cm}$ panel specimens in a single pass of the camera along the track.
Fig. 2 Diagram of the track system used in the exposure tests

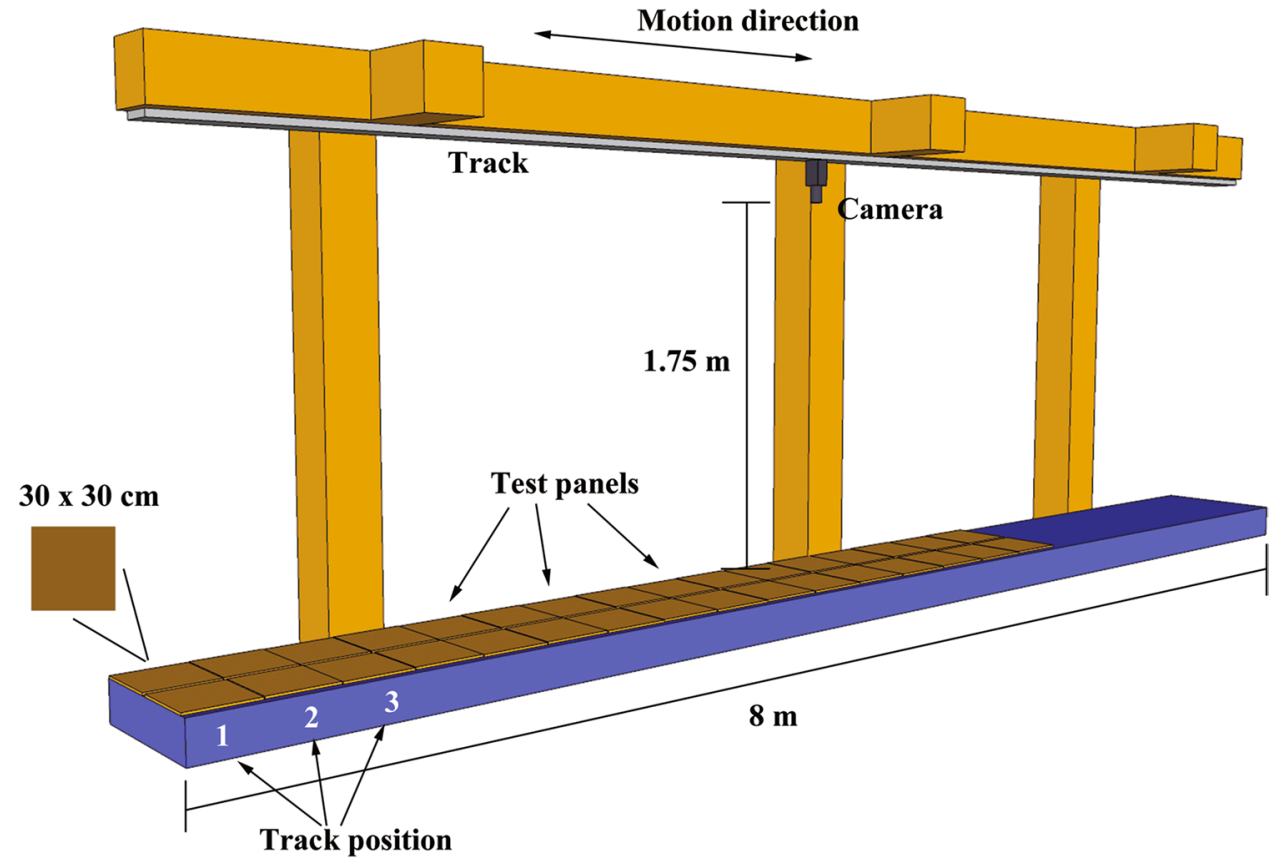




\subsubsection{Climate control system}

To rapidly induce checking, specimens were first conditioned in a warm and humid environment $\left(30{ }^{\circ} \mathrm{C}\right.$ and $90 \%$ RH set points) then exposed to harsh, yet realistic warm and dry (approximately $30{ }^{\circ} \mathrm{C}$ and $26 \% \mathrm{RH}$ ) environmental conditions in a climate enclosure built around the track. The enclosure consisted of a sealed plastic curtain enveloping the entire track system, providing a greater degree of environmental control than available through standard climate control in the laboratory (Fig. 3).

The climate inside the sealed area was maintained by two $1500 \mathrm{~W}$ space heaters (DeLonghi model 2507) placed along the track at approximately 2 and $4 \mathrm{~m}$ from each end (as shown in Fig. 3), and a dehumidifier (Frigidaire model FAD504DUD) with a nominal capacity of $23.7 \mathrm{dm}^{3}$ water per day, placed at one end of the track. The heaters were set to "medium", while the humidifier was set to run continuously at its full capacity. The air was kept in motion with a household box fan set at its "medium" setting placed near

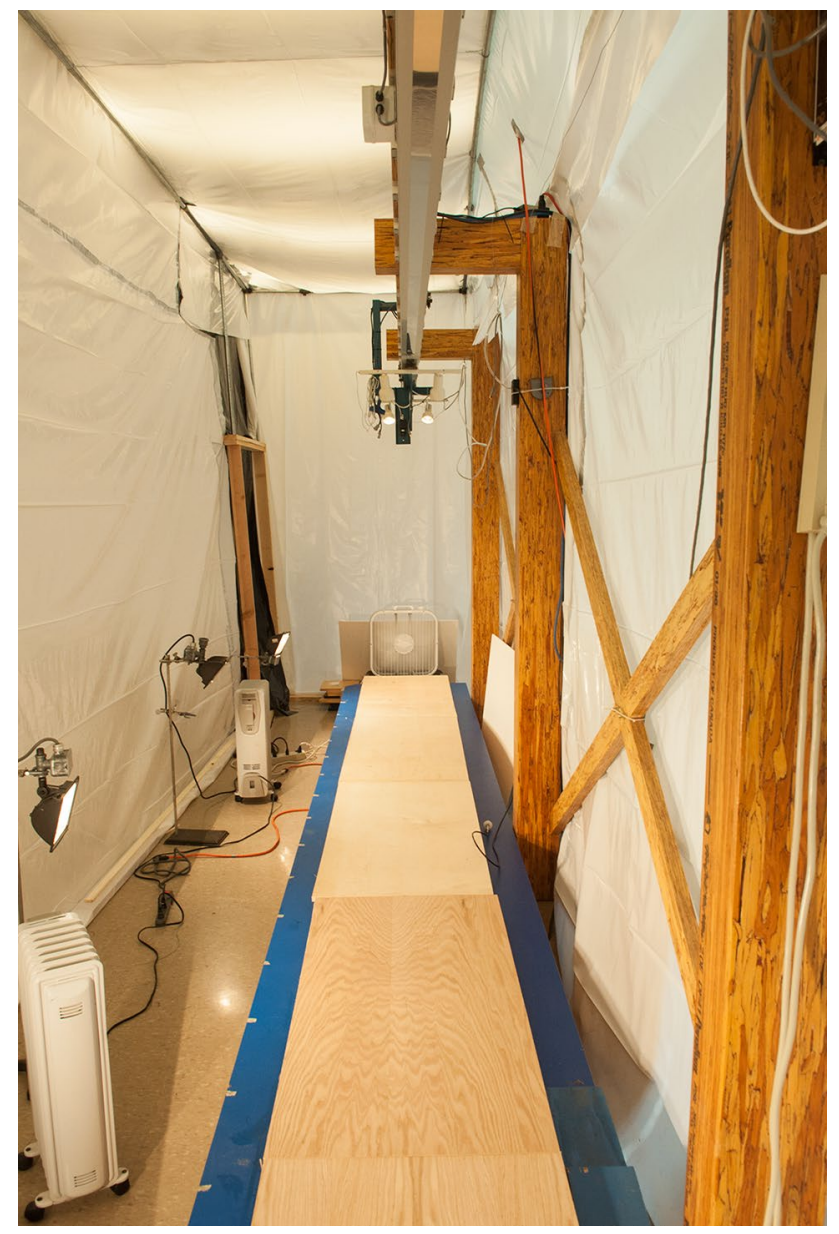

Fig. 3 Elements of the climate control system within enclosure (without specimens) the starting position of the track system and level with the test specimens. Four $50 \mathrm{~W}$ halogen lamps used to illuminate the track also contributed to heating the enclosure and panel surfaces.

The climate in the enclosure was monitored with Vaisala Humidity and Temperature Transmitter HMT 330 positioned at the midpoint of the track system, approximately $5 \mathrm{~cm}$ from the edge of the observation area, and approximately $5 \mathrm{~cm}$ above the panels' surface.

This climate control system enabled maintaining conditions of $30.6 \pm 0.7{ }^{\circ} \mathrm{C}$, and relative humidity of $26.3 \pm 1.8 \%$ over more than $8 \mathrm{~h}$. For these conditions the equilibrium moisture content (EMC) in solid wood will vary from 5.1 to 5.7\% (Simpson 1998).

\subsubsection{Optical measurement system}

The optical measurement system consisted of a monochrome five-megapixel $(2448 \times 2048$ pixel $)$ camera with a $1.69 \mathrm{~cm}$ CMOS sensor (Allied Vision Technologies Grasshopper2) and a $23 \mathrm{~mm}$ lens (Schneider Kreuznach Xenoplan 1.4/23) mounted on an $8 \mathrm{~m}$ horizontal linear positioning track moving over a stage. The track system was powered by a Parker Daedel 204,000 Series motor and controlled by a Parker Compumotor SX-6 Drive, connected via a RS-232 serial interface to a PC. Position control of the track and hence, placement of the camera, could be incremented in steps as precise as $0.006 \mathrm{~mm}$. The camera was mounted so that the front of the lens was $1.75 \mathrm{~m}$ above the specimen surface. At this working distance, two side-by-side specimens could be entirely enclosed in the field of view of the camera. The resulting resolution of digital images was $0.26 \mathrm{~mm} /$ pixel. The Schneider Kreuznach Xenoplan 1.4/23 lens specifications report the maximum distortion at the edges of the lens is within $-1 \%$ of the true dimension for working a distance of $1.16 \mathrm{~m}$ (negative value indicates barrel distortion) (Jos. Schneider Optische Werke GmbH 2008). In this project, the working distance was $1.75 \mathrm{~m}$, so even smaller distortions were expected. Therefore, distortion was considered a negligible source of error in this project.

The system was set to capture a series of images of test panels along the track (pair-wise). These images for each specimen pair were taken every 10 min over the exposure test duration (240 min). The first series captured at the beginning of the exposure test provided reference images for each individual specimen. Images of specimens captured throughout the duration of the test were compared to the first image (at time 0) to obtain changes in full-field strain and displacements on the panel surfaces at each 10 min interval.

The DIC software used to analyze the images was ARAMIS version 5.4.3 (GOM, mbH 2004). The accuracy and precision of the DIC analysis depends not only on the robustness of the software and quality of the optical hardware but 
on many other factors including lighting, contrast and the relative size of the speckle pattern (a light application of black and white matte spray paint used to create identifiable points on the panel surface), parameters of the analysis (notably, the facet size, or the size of the target area, defined in square pixels used to identify a point between images, and the step size, or the distance between the centers of adjacent facets) and the skills of the operator. Therefore, the accuracy and precision are best assessed in the actual setup on specimens of the same characteristics as those to be used in the test. For this purpose, a series of five images of one specimen were taken in rapid succession (within, approximately, $2 \mathrm{~s}$ ), so that no deformation due to shrinkage was expected. Consequently, all displacements or strains reported by the DIC software were considered error or noise in this 'testing' phase. The standard deviation of the displacement noise reported for all reference points on the measured surface provided a convenient measure of precision of the measurements in millimeters, while the deviation of the mean value of the noise from the expected displacement value (zero) was considered a measure of accuracy. A typical outcome of such test series is shown in Fig. 4, where points represent the mean of the displacement noise measured in direction perpendicular to the grain and the error bars represent the standard deviations.

This method was used before the test series to determine parameters of the DIC algorithm that minimize the noise, and then at the beginning of each exposure test run to confirm that the precision was maintained as the speckle patterns and light conditions varied slightly. The set of DIC parameters used in this study (facet size of 9 pixels and step size of 7 pixels) determined using this procedure resulted in measurement accuracy of $\pm 0.0016 \mathrm{~mm}$ and precision of $\pm 0.03 \mathrm{~mm}$, or $15 \%$ of the smallest qualified check width $(0.2 \mathrm{~mm})$.

In the course of one exposure test, the optical measurement system generated 25 stage data sets for each specimen at $10 \mathrm{~min}$ intervals. These stage outputs capture the state of the panel (in terms of displacements and strains at each surface point) at each time interval. Every numerical ARAMIS stage output file provided Cartesian coordinates, components of the displacement vectors, and components of surface strains tensor for each of about 25,000 reference points in the region of interest of the examined panel. A sample strain map output from optical measurement software in Fig. 5a shows apparent positive strain spikes along the check edges as dark lines in the middle of the panel. Dark areas on the edges of the panel indicate high negative strain or shrinkage where veneers were debonded from the core materials.

\subsubsection{Check detection and measurement procedure}

Although the presence and location of checks on such strain maps are reasonably obvious for a human inspector, it is important to note that the standard optical measurement software packages based on DIC provide no tools to distinguish between checks and other unrelated spikes in strains, and no direct measurement of check position, count, size, or checking severity. Therefore, a custom software procedure was designed to calculate these characteristics from the ARAMIS output data. The analysis was focused on normal strain components across the grain on the surface of the decorative veneers $\left(\varepsilon_{\mathrm{xx}}\right)$. The principal steps of the analysis were:

1. Automatic preliminary identification of signature apparent strain peaks above the threshold of $2.5 \%$ as possible checks. This threshold level was established in test trials on more than 20 specimens prior to this pilot study, where the potential checks identified with the threshold were compared with actual checks on the test surfaces detected by visual inspection and measured manually. A comparison of check locations detected by the program to the visual output from the ARAMIS software is shown in Fig. 5.

2. Determination of check positions and lengths. Cartesian coordinates of the points detected as potential checks in step 1 were examined for continuity along the
Fig. 4 Plot of 5 consecutive means (points) and standard deviations (error bars) of the full-field displacement noise measured on an undeformed specimen. The data were used for the assessment of the accuracy (mean) and precision (standard deviation) of the optical measurement system for the specific experimental setup

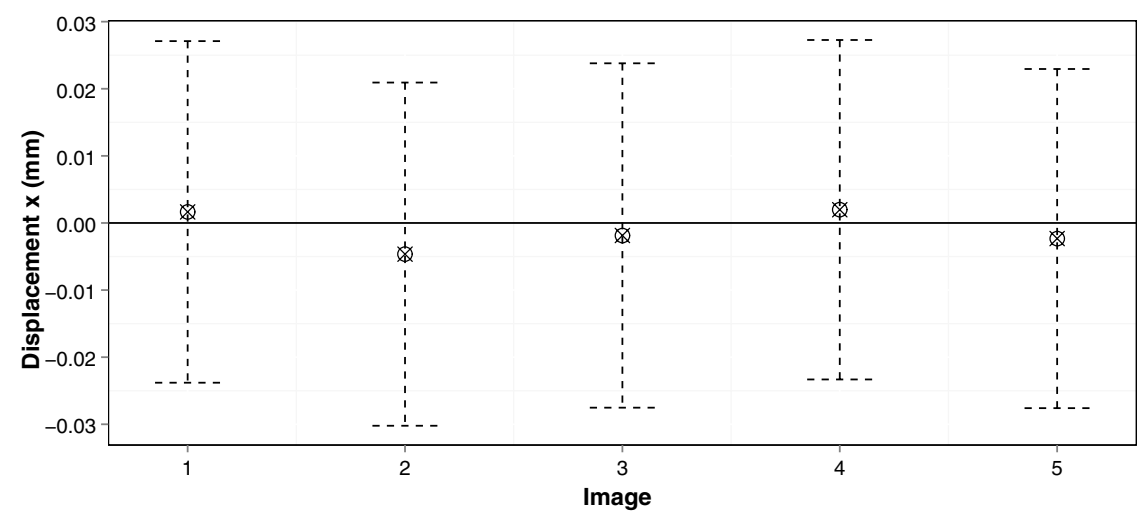



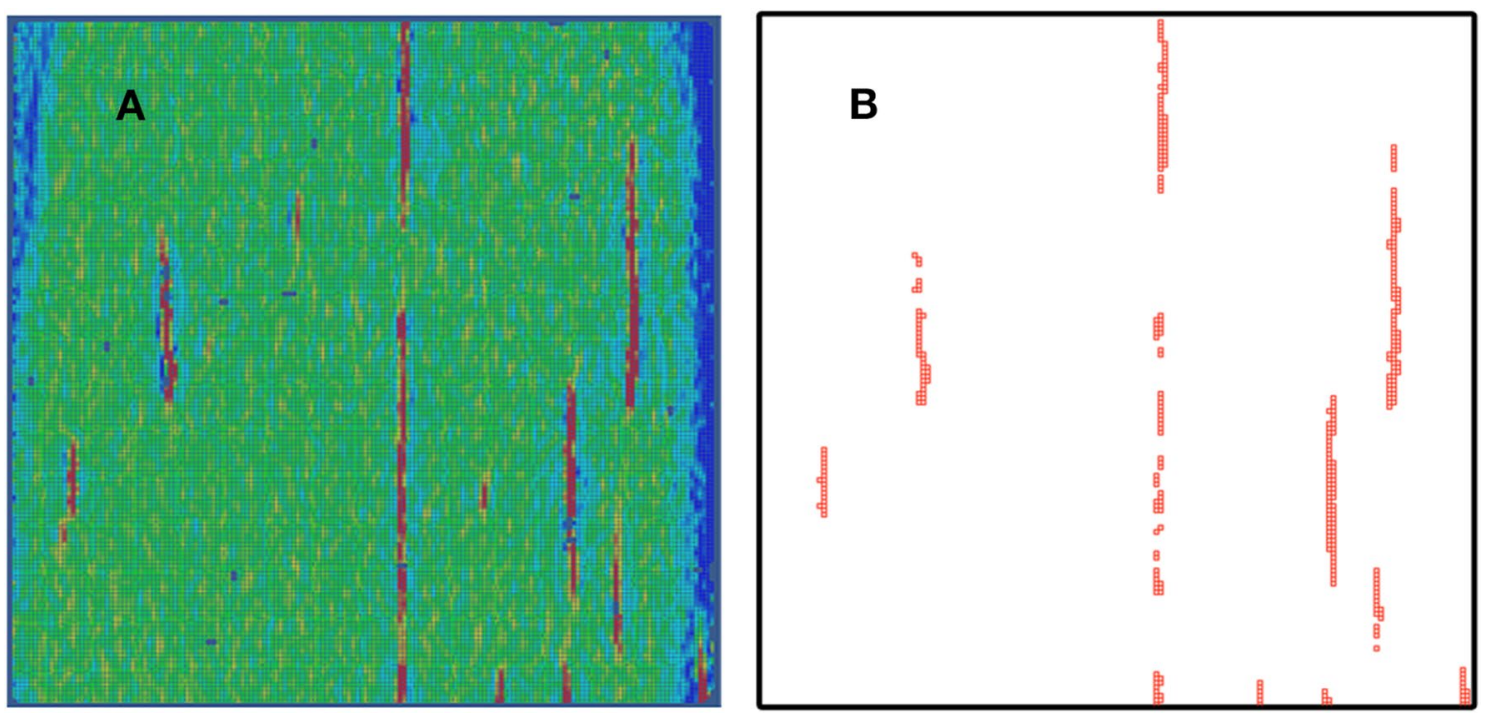

Fig. 5 Image output of DIC software (a) compared to image output from custom check characterization software (b)

grain direction $(y)$. Next, the $x$ displacement increments between points with contiguous $x$ coordinates were analyzed to determine check width and eliminate points indicating widths below the $0.2 \mathrm{~mm}$ threshold. The number of remaining contiguous point groups was used to calculate check count within the panel, while extremes of contiguous $y$ coordinates within each group were used to estimate individual check lengths. Check areas were calculated from individual check lengths and the average width of the check.

3. Determination of check intensity characteristics. The individual check dimensions were used to calculate cumulative check characteristics for each panel: check count (total number of checks) on the surface at given stage, minimum, maximum and average check dimensions, total check area and the check density (CD) as defined in Eq. 1. These cumulative characteristics were then used to trace the check development over the duration of the exposure tests and to compare the propensity for checking between tested materials (i.e., combinations of core type, adhesive, etc.).

\subsection{Test procedures}

Two subsets of data from a study that examined the effect of panel manufacturing factors on checking in maple veneered plywood panels (Burnard 2012) are used here to illustrate the abilities and efficiency of the new optical method. The first subset are individual panels selected to highlight the system's ability to provide insight into patterns of check development over time. The second subset are 8 replicates each of two groups of test panels (16 total panels). The two groups differ only in the type of face veneer used for their construction. The groups are, Group A: sliced $0.508 \mathrm{~mm}$ face veneer oriented with lathe checks facing away from the core (loose-side out), using urea-formaldehyde adhesive, and MDF core; and Group B: sliced $0.564 \mathrm{~mm}$ face veneer oriented with lathe checks facing away from the core, using urea-formaldehyde adhesive, and MDF core.

The test panels measured $30 \times 30 \mathrm{~cm}$, and were assembled from pre-cut cores and veneers provided by a cooperating company. Adhesives were applied with a laboratory scale spreader, with a target spread rate of $177 \mathrm{~g} / \mathrm{m}^{2}$ (target, $15.9 \mathrm{~g}$ per glue line; measured: mean: $15.9 \pm 1.3 \mathrm{~g}$ per glue line). Prior to pressing, all panel components (aside from adhesive) were conditioned at ambient lab environment levels for seven days before assembly: temperature $21.4 \pm 0.6^{\circ} \mathrm{C}$; relative humidity $31.3 \pm 5.9 \%$. The pressing procedure was as follows: after adhesive spreading and assembly, panels were cold pressed at $0.93 \mathrm{GPa}$ for $5 \mathrm{~min}$, then hot pressed for 2 min at $0.93 \mathrm{GPa}$ with the top platen set at $113^{\circ} \mathrm{C}$.

All samples were stored in a chamber with climate set points of $20^{\circ} \mathrm{C}$, and $65 \%$ relative humidity (solid wood equilibrium moisture content of $12 \%$ ) for at least $72 \mathrm{~h}$ prior to beginning the test procedure.

The overall test procedure consisted of the following major steps:

1. In order to generate aggressive moisture gradients and increase the likelihood of checking, prior to the exposure tests all specimens were conditioned in a high humidity climate $\left(30{ }^{\circ} \mathrm{C}\right.$ and $90 \%$ relative humidity; solid wood equilibrium moisture content of $20 \%$ ) for $24 \mathrm{~h}$.

2. The test climate control in the enclosure was turned on 45 min prior to the test to warm the chamber and reduce the relative humidity. 
3. Up to $48,30 \times 30 \mathrm{~cm}$ test panels were placed in random order on the track system surface with their surface grain direction parallel to the direction of camera motion on the overhead track. This step took approximately $5 \mathrm{~min}$.

4. Five initial reference images of the test panels were captured for assessment of precision and accuracy.

5. During the 4-h exposure tests, the track control and image acquisition captured series images of pairs of panels arranged along the track once every $10 \mathrm{~min}$. Temperature and relative humidity parameters near the panel surfaces were recorded at the same time intervals.

6. Data processing and analysis (e.g., strains, check counts, assessing check width, etc.) were conducted after completion of the test series as described previously.

\section{Results}

As stated previously, objective criteria for surface features that qualify as a check were established as separations of the grain that are at least $0.2 \mathrm{~mm}$ wide and at least $1.0 \mathrm{~mm}$ long by manual examination of panels with existing checks that caused complaints from panel manufacturer's customers. These criteria were used as threshold values in an automated check characterization algorithm utilizing output from digital image correlation software and recorded check counts, check widths, check lengths, check areas, and a newly developed check severity index, $\mathrm{CD}$, which allows observers to quickly and objectively compare checking between panels. Furthermore, these values are available in a time series which allows researchers to monitor check development over time.

A specimen handling system was developed that allowed concurrent examination of up to $48,30 \times 30 \mathrm{~cm}$ test specimens over a user-selected period of time and at user-selected intervals. This system provided rapid data collection that was processed first in DIC software, then by the check detection and measurement system, which processed data in bulk. In this study, the average area examined for checks on each panel was $768 \pm 33 \mathrm{~cm}^{2}(85 \pm 4 \%$ of the final panel surface area).

Check detection was based on identifying signature strain patterns described in Kang et al. (2011). The preliminary selection tool based on the strain threshold value (2.5\%) served as a data reduction step that reduced the number of locations to search for checks on the panel surface. This pre-selection step may not be necessary as the computing power continues to rapidly increase. This step will work properly only when the strain threshold level is selected in a way that does not eliminate checks that might have been qualified based on the displacementbased threshold.

\subsection{Check detection over time}

With the data from the check detection system, distinct patterns in check development were detected. These patterns could be categorized into four groups: panels that reached their peak CD early in the test period (Early Peak in Fig. 6); panels that reached an apparent level of equilibrium during the test period (Equilibrium in Fig. 6); panels that exhibited significant growth late in the test period (Late Growth in Fig. 6); and panels that grew at a steady rate from the onset of checking to the end of the test period (Steady Growth in Fig. 6). One practical implication of these patterns for manufacturers is an indication of how 'robust' the panel construction is to changes in ambient conditions; plywood manufacturers would likely desire a panel that would react slowly (if at all) to changes in temperature and humidity.

\subsection{Comparing checking between panel groups}

The two panel groups had similar checking patterns: little or no checking in most panels, and one panel with more severe checking (Fig. 7). In these groups the average amount of checking was heavily influenced by the panels with little to no checking, while the panel with uncharacteristically high CDs were outliers within their groups. Outliers such as these make summarizing checking amongst groups of panels subject to possible misinterpretation, but may help to explain apparent contradictions in previous research such as the effect of lathe-check orientation on check formation (c.f., Cassens and Leng 2003; Leavengood et al. 2011), and the challenges manufacturers have in addressing complaints related to checking.

Group A reached its peak mean CD (the peak of the averaged values by stage) of $48.6 \mathrm{~mm}^{2} / \mathrm{m}^{2}$ at the 210 th minute of the test period, while Group B reached its peak mean CD of $33.2 \mathrm{~mm}^{2} / \mathrm{m}^{2}$ at the 240th minute (Table 1). The number of panels with detectable checks also differed between groups: there were five panels with detectable checks in Group A, and only two panels with detectable checks in Group B (Table 1). The greatest CD recorded in all 16 test panels was $229 \mathrm{~mm}^{2} / \mathrm{m}^{2}$ on a panel in Group B at the 220th minute.

In Table 2, the maximum observed value for a variety of check description statistics are provided to illustrate the variety of data produced by the method.

\section{Conclusion}

An automated method for detecting and measuring surface check characteristics and their development over the exposure time has been successfully applied in this project. This system also allowed identification and measurement of checks as narrow as $0.2 \mathrm{~mm}$ wide with a precision of 

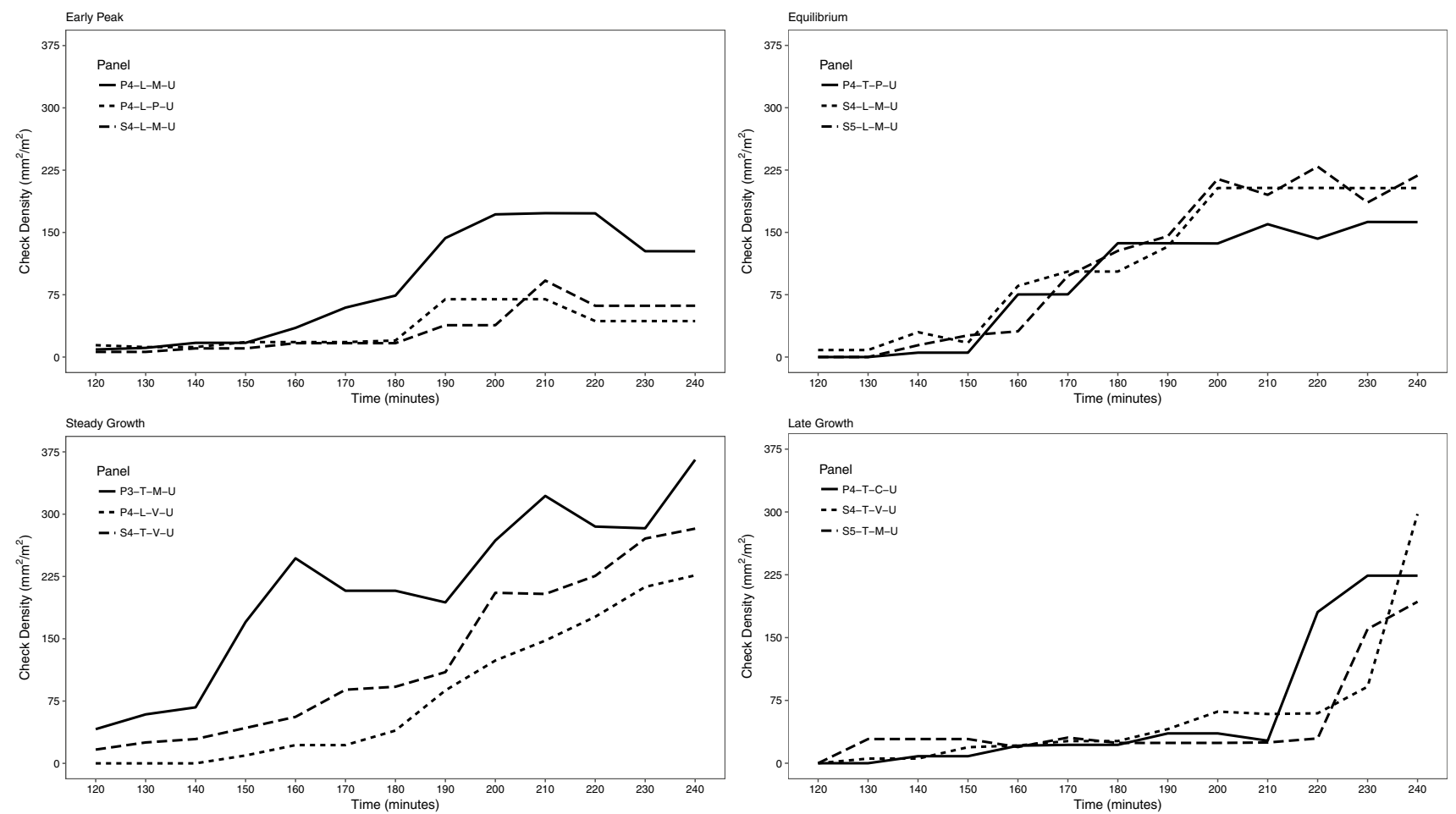

Fig. 6 Four groups of check development patterns visible in individual panels. Three individual reference panels were selected for each group to demonstrate that there is variation within each group. Legend key: P3 = Peeled veneer, $0.706 \mathrm{~mm}, \mathrm{P} 4=$ Peeled veneer,

$0.604 \mathrm{~mm}, \mathrm{~S} 4=$ Sliced veneer, $0.564 \mathrm{~mm}, \mathrm{~S} 5=$ Sliced veneer, $0.508 \mathrm{~mm} ; \mathrm{L}=$ Lathe checks out, $\mathrm{T}=$ Lathe checks in; $\mathrm{C}=$ Combination core, $\mathrm{M}=\mathrm{MDF}, \mathrm{P}=$ particleboard, $\mathrm{V}=$ plywood veneer core; $\mathrm{U}=$ Urea formaldehyde adhesive

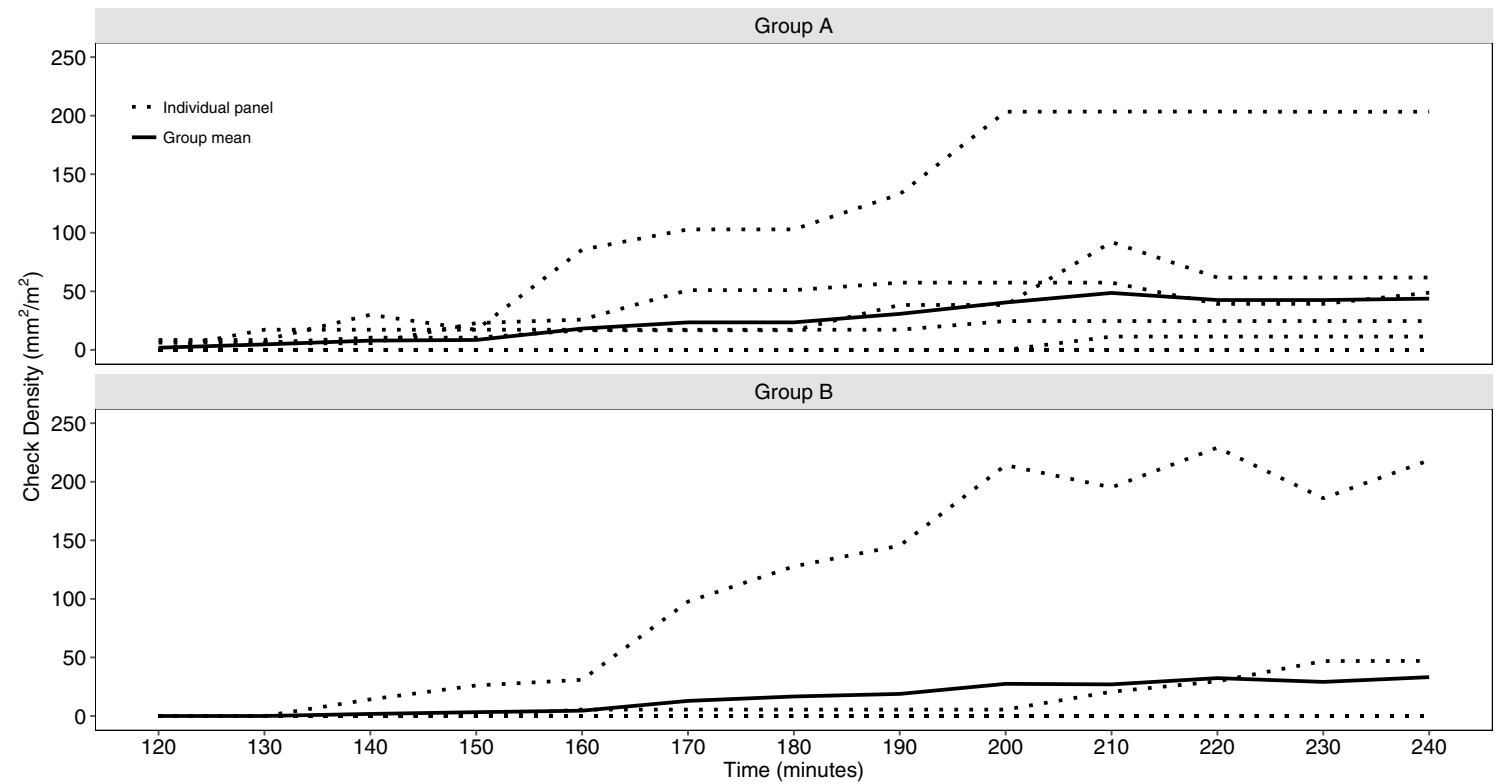

Fig. 7 Check progression for two groups of panels that varied only in the type of face veneer utilized (Group A: Sliced 0.564 mm, Group B: Sliced $0.508 \mathrm{~mm}$ ) 
Table 1 Summary of key statistics for panel groups A and B

\begin{tabular}{llllll}
\hline $\begin{array}{l}\text { Panel } \\
\text { Group }\end{array}$ & $\begin{array}{l}\text { Peak } \\
\text { mean CD } \\
\left(\mathrm{mm}^{2} /\right. \\
\left.\mathrm{m}^{2}\right)\end{array}$ & $\begin{array}{l}\text { Peak } \\
\text { mean CD } \\
\text { minute }\end{array}$ & $\begin{array}{l}\text { Peak CD } \\
\left(\mathrm{mm}^{2} / \mathrm{m}^{2}\right)\end{array}$ & $\begin{array}{l}\text { Peak } \\
\mathrm{CD} \\
\text { minute }\end{array}$ & $\begin{array}{l}\text { No. of } \\
\text { panels with } \\
\text { checks }\end{array}$ \\
\hline A & 48.6 & 210 & 204 & 240 & 5 \\
B & 33.2 & 240 & 229 & 220 & 2 \\
\hline
\end{tabular}

Table 2 Summary statistics of check data for each panel group. Each observation is the maximum observed within the group, and may come from different panels within the group

\begin{tabular}{lll}
\hline Statistic & Group A & Group B \\
\hline Total check area & $13.53 \mathrm{~mm}^{2}$ & $4.30 \mathrm{~mm}^{2}$ \\
Largest individual check & $1.88 \mathrm{~mm}^{2}$ & $0.79 \mathrm{~mm}^{2}$ \\
Average check area & $3.68 \mathrm{~mm}^{2}$ & $2.15 \mathrm{~mm}^{2}$ \\
Longest check & $10.92 \mathrm{~mm}$ & $5.46 \mathrm{~mm}$ \\
Total check length & $45.24 \mathrm{~mm}$ & $10.92 \mathrm{~mm}$ \\
Average check length & $11.70 \mathrm{~mm}$ & $0.39 \mathrm{~mm}$ \\
Widest check & $1.01 \mathrm{~mm}$ & $0.50 \mathrm{~mm}$ \\
Number of checks & 9 & 5 \\
\hline
\end{tabular}

$0.03 \mathrm{~mm}$ ( $15 \%$ of the minimum identifiable width). The automated measurements are reliable, repeatable, and the method offers unprecedented efficiency and detail. It allows for more sophisticated analysis of both check severity and check development over time than was previously possible. This is a substantial advantage compared to measurements conducted by a human investigator, which are more subjective and may vary when the same panel is examined multiple times.

The efficiency of this method was achieved by automation, the measurement systems capacity to monitor multiple test specimens at once, and accelerating check formation by conducting tests in a low-humidity chamber after raising the moisture content of the panels in a high temperature high humidity chamber prior to testing.

Further refinement of this method may include automation of the digital image correlation analysis of the images through batch processing scripts supported in newer versions of the ARAMIS software. Additionally, comprehensive guidelines should be proposed for selecting system parameters based on field of view, material characteristics (such as ultimate tensile strength of the surface veneer of a decorative panel), test parameters (such as physical load applied or experienced, qualification size for the crack to be detected), camera and lens characteristics that estimate system settings such as the strain threshold, facet, and step size.

The strain threshold criterion used to pre-select areas to be analyzed for suspected checks should be selected with caution. The purpose of the procedure was to reduce the machine time by limiting the number of points to be examined for check characteristics, however, when set improperly (too high) it may impact the number of checks found by improperly eliminating those that might pass the width criterion. While the $2.5 \%$ level was carefully selected for this study, in future studies the proper level of the threshold should be investigated individually for each setup.

Other uses of this method include detecting checks, splits, or cracks in other composite materials or in solid lumber, or to examine coating performance and failures. Surfaces that should not have a speckle pattern applied (e.g., art or other cultural heritage items) present a limitation for this technique.

Acknowledgements This project was funded by the United States Department of Agriculture Special Grant program for Wood Utilization Research with generous contributions from the members of the Western Hardwood Plywood Producers-Columbia Forest Products, Murphy Plywood, Roseburg Forest Products, States Industries and Timber Products Company.

Open Access This article is distributed under the terms of the Creative Commons Attribution 4.0 International License (http://creativeco mmons.org/licenses/by/4.0/), which permits unrestricted use, distribution, and reproduction in any medium, provided you give appropriate credit to the original author(s) and the source, provide a link to the Creative Commons license, and indicate if changes were made.

\section{References}

Alam SY, Loukili A, Grondin F (2012) Monitoring size effect on crack opening in concrete by digital image correlation. Eur $\mathbf{J}$ Environ Civ Eng 16(7):818-836. https://doi.org/10.1080/19648 189.2012.672211

ASTM International (1999) ASTM Standard D9, Standard Terminology Relating to Wood and Wood-Based Products. ASTM International, West Conshohocken

Batey TEJ (1955) Minimizing face checking of plywood. Forest Prod J 5(10):277-285

Burnard M (2012) Key Factors Influencing Checking in Maple Veneered Decorative Hardwood Plywood. Oregon State University. Corvallis, OR, USA. Master's thesis

Cassens D, Leng Y (2003) Face check development in veneered furniture panels. Forest Prod J 53(10):79-86

Christiansen AW, Knaebe M (2004) Diagnostic guide for evaluating surface distortions in veneered furniture and cabinetry. Technical Report. US Forest Service Forest Products Laboratory Madison, WI, USA

Fan W, Qiao P (2011) Vibration-based damage identification methods: a review and comparative study. Struct Health Monit 10(1):83-111. https://doi.org/10.1177/1475921710365419

Felix S, Morlier P (1992) Modeling of stresses and strains in a piece of wood under drying. Holzforschung 46(5):369-378

Forbes C (1997) Understanding and minimizing veneer checking on furniture panels. North Carolina State University. Raleigh: North Carolina State University. Online (https://content.ces. ncsu.edu/understanding-and-minimizing-veneer-checking-onfurniture-panels) Accessed March 27, 2018 
Gilmore R, Hanover S (1990) Suggestions for preventing or minimizing veneer checking. North Carolina State University. Raleigh, NC: North Carolina State University. Online https://research. cnr.ncsu.edu/blogs/wpe/publications/suggestions-for-preventing -or-minimizing-veneer-checking/ Accessed March 27, 2018

GOM, mbH (2004) Aramis version 5.4.3. [Software application]. GOM, mbH, Braunschweig

Helm JD (2008) Digital image correlation for specimens with multiple growing cracks. Exp Mech 48(6):753-762. https://doi. org/10.1007/s11340-007-9120-2

Holcombe RA (1952) Surface checking in furniture panels. J For Prod 2(5): 122-127

Jos. Schneider Optische Werke GmbH (2008) Schneider Kreuznach Ruggedized Lens [Product specification]. Online: https://schne iderkreuznach.com/download_file/force/574/1085. Accessed April 9, 2018

Kang H-Y, Muszynski L, and Milota MR (2006) Optical measurement of non-uniform drying strains and drying check formation in refractory species. In: Proceedings of FPS conference on quality drying for the 21st century, Bellingham, pp. 15-17

Kang H-Y, Muszyński L, Milota M, Kang C, Matsumura J (2011) Preliminary tests for optically measuring drying strains and check formation in wood. J Fac Agric Kyushu Univ 56(2):313-316

Leavengood S, Funck JW, Reeb EW (2011) A note on face veneer checking in maple plywood. Int Wood Prod J 2(2):120-123

Mårtensson A, Svensson S (1997) Stress-strain relationship of drying wood. Part 2: verification of a one-dimensional model and development of a two-dimensional model. Holzforschung 51(6):564-570

Ormarsson S, Dahlblom O, Petersson H (1999) A numerical study of the shape stability of sawn timber to moisture variation. Part 2. simulation of drying board. Wood Sci Technol 33:407-423

Réthoré J, Hild F, Roux M (2008) Extended Digital image correlation with crack shape optimization. Int J Numer Meth Eng 73(2):248 272. https://doi.org/10.1002/nme.2070

Schramm A (2003) A complete guide to hardwood plywood and face veneer. Purdue University Press, West Lafayette

Simpson WT (1998) Equilibrium moisture content of wood in outdoor locations in the United States and worldwide. Research Note. Forest Products Laboratory, Madison

Suchsland O (2004) The swelling and shrinking of wood: a practical technology primer. Forest Products Society, Madison

Sutton MA, Chao YJ (1988) Measurement of strains in a paper tensile specipmen using computer vision and digital image correlation. TAPPI J 71(3): 173-175

Svensson S, Martensson A (2002) Simulation of drying stresses in wood: Part II Convective air drying of sawn timber. Holz Roh Werkst 60(1):72-80

Publisher's Note Springer Nature remains neutral with regard to jurisdictional claims in published maps and institutional affiliations. 